Historic, Archive Document

Do not assume content reflects current scientific knowledge, policies, or practices. 



\section{Peony and Iris Bargains}

In order to reduce the number of varieties in both catalogue and gardens, we have determined to offer the following well-known standard Peonies and Iris at greatly reduced prices until stock of same is exhausted. Orders will be filled in rotation.

\section{PEONIES}

Agnes Mary Kelway.\$o 50 L'Indispensable.... .\$o 35 Armandine Mechin. . Auguste Villaume... Belle Mauve.

Bunch of Perfume... Claude Gellée...... . . Dr. Bretonneau..... Grandiflora lutescens Graziella........... Limosel.
75

75

200

75

I 00

35

35

I 00

I 00
Lucrece.

Meissonier

Mme. Lemoinier. . . .

Mons. Bastien le Page

Souv. de l'Exposition

de Bordeaux...... I oo

Ville de Nancy..... . 50

Virgo Maria........ 50

Zephyrus......... 50

\section{IRIS}

Abdul Aziz. . . . . . . \$o Agnes.

Blue Jay

Bridesmaid .

Chelles.

Comte de St. Clair. . Cordelia.

Edward Simmons....

Foster's Yellow..... .

Frederick.

Fro.

Glory of Hillegom. . Glow.

Gracchus.

Innocenza......... .

Kathleen

Kharput

Maritana
I 5 Mary Minanelle.... \$o I5

I 5 Miss E. Eardley..... 25

35 Mme. Chereau..... I. I5

25 Monhassan........ 25

I 5 Nokomis........... 35

20 Oriental......... 75

25 Ossian........... 25

I5 Patience.......... 50

I5 Penge.......... 25

I5 Pfauenauge. . .... 50

35 Plumeri.......... I5

I5 Sybil........... I5

35 Tecumseh.......... 25

I5 Thorbeck......... I5

I5 Trautlieb.......... 25

I5 Trojana.......... I5

I5 Trojana magnifica... 20

I 5 Walter Reuthe..... 20

Terms: Cash with order. No order accepted for less than $\$ 1$.

\section{MOVILLA GARDENS}


